\title{
Towards spin torch experiments and artificial reaction centers
}

\author{
Karthick Babu Sai Sankar Gupta
}

Published online: 20 November 2009

(C) The Author(s) 2009. This article is published with open access at Springerlink.com

\begin{abstract}
Harvesting solar energy for human use has long been a cherished dream of scientists. Especially when unprecedented potentials of biological systems are explored, they could shift the paradigm in the field of development of bio-electronic devices. Hence artificial photosynthetic reaction centers can be potential 'photovoltaic cells at molecular dimensions'. Understanding the chemical environment, structure and dynamics plays an important role towards the effort of achieving an 'artificial reaction center'. Let us see how S. Karthick Babu, an EMBO student describes how 'spin torch' experiments are designed to chase this dream.
\end{abstract}

Keywords Reaction center - Photo-CIDNP NMR . Photosynthesis

Artificial photosynthetic reaction centers are manmade systems or devices to mimic nature's most sophisticated engines i.e. the solar energy storage systems as found in photosynthetic organisms. The

K. B. Sai Sankar Gupta ( $\square)$

SSNMR, LIC, Leiden University, Leiden,

The Netherlands

e-mail: skarthickbabu@gmail.com unparalleled promise that the reaction centers has, that ranges from biofuel cells to photosystem II based biosensors for detection of photosynthetic herbicides (Dimitrios et al. 2008), and effective and rapid environmental screening of various pollutants, pesticides etc. makes it a hot topic of research. Hence to better design such a molecular shuttle, it's imperative to understand the molecular structure and chemical environment. In such a system, amongst other methods for determination of structure and dynamics such as crystallography and nuclear magnetic resonance (NMR), solid-state NMR has developed into a feasible technique to approach biological structures at the atomic resolution (Baldus 2007).

The most challenging problem in solid state NMR is its low sensitivity and selectivity. A method to overcome this problem may be photo-chemically induced dynamic nuclear polarization (photo-CIDNP), magic angle spinning (MAS) NMR leading to high nuclear polarization of a factor of more than 10,000 above the Boltzmann equilibrium (Jeschke and Matysik 2003; Prakash et al. 2005). This high nuclear polarization is produced in light-induced radical pairs. Currently, the effect is limited to natural photosynthetic reaction centers (RCs), however, it is expected that also artificial RCs will be used in the near future. The aim of the project is to use the enormous nuclear polarization to explore the surroundings protein pockets, protein surfaces and membrane surfaces by transfer of photo-CIDNP polarization. 


\section{Model system}

\subsection{The reaction center of Rhodobacter sphaeroides}

The RCs of Rhodobacter sphaeroides (Rb. sphaeroides) is a transmembrane protein complex made of three major polypeptides, H, L and M (for heavy, medium and light). The $\mathrm{L}$ and $\mathrm{M}$ subunits contain five transmembrane $\alpha$-helices, which are packed together in a nearly symmetrical way. Subunit $\mathrm{H}$ is more globular in shape and is located mainly in the cytoplasmic side of the membrane. The $\mathrm{L}$ and $\mathrm{M}$ subunits bind the cofactors. Four molecules of bacteriochlorophyll $a(\mathrm{BChl} a)$, two molecules of bacteriopheophytin $a$ (BPhe $a$ ), two ubiquinone-10 molecules (Q), a non-heme iron $\left(\mathrm{Fe}^{2+}\right)$ and a caroteniod molecule (C) form the cofactors of the RCs protein. The arrangements of cofactors are shown in Fig. 1.

They are arranged in two nearly symmetric branches, the "active" A-branch and the "inactive" B-branch. Two BChls form a tightly interacting dimer called the "Special Pair" (P). On either side of the special pair an additional $\mathrm{BChl}$ molecule is located, known as the accessory $\mathrm{BChl}\left(\mathrm{B}_{\mathrm{A}}\right.$ and $\left.\mathrm{B}_{\mathrm{B}}\right)$. The two BPhe $(\Phi)$, ubiquinones-10, $\left(\mathrm{Q}_{\mathrm{A}}\right.$ and $\left.\mathrm{Q}_{\mathrm{B}}\right)$. Finally, the non-heme $\mathrm{Fe}^{2+}$ ion is located in the center of the two branches near the cytoplasmic side of the membrane. The tenth cofactor, the carotenoid molecule (C), breaks the overall symmetry of the cofactor arrangement and is located near $\mathrm{B}_{\mathrm{B}}$. In the RCs of $R b$. sphaeroides $\mathrm{R} 26$, a mutant strain, no carotenoid

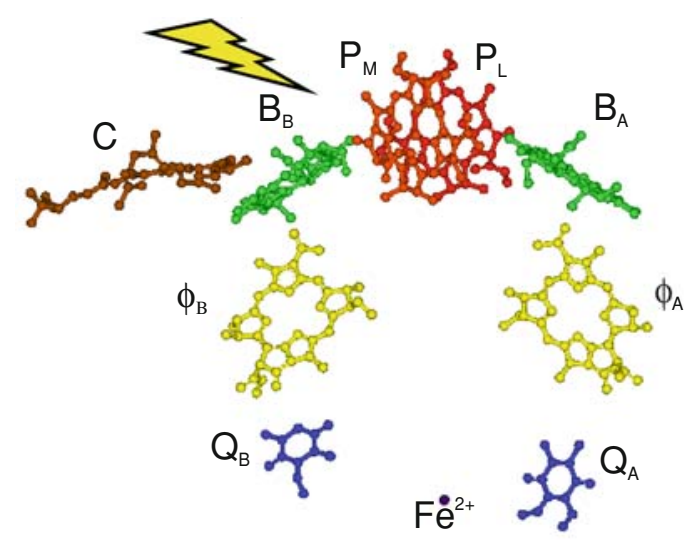

Fig. 1 Bacterial reaction center of Rhodobacter sphaeroides (1M3X.pdb) molecule is present. Despite symmetry in the structure, the electron-transfer pathway in the RCs is asymmetric. The electron transfer proceeds almost exclusively via the A branch. The reason for this functional asymmetry is still being explored by electron paramagnetic resonance (EPR) techniques namely by continuous wave or pulsed electron nuclear double resonance (ENDOR) techniques.

After photochemical excitation of $\mathrm{P}$ to $\mathrm{P} *$, one electron is emitted which is transferred to the primary electron acceptor $\Phi_{\mathrm{A}}$ within $3 \mathrm{ps}$, forming the radical pair state $\mathrm{P}^{+\bullet} \Phi_{\mathrm{A}}^{-\bullet}$. The $\Phi_{\mathrm{A}}^{-\bullet}$ anion radical decays in about $200 \mathrm{ps}$ and transfers an electron to the ubiquinone $\mathrm{Q}_{\mathrm{A}}$. The electron subsequently moves from $Q_{A}$ to $Q_{B}$ in $200 \mu$ s reducing $Q_{B}$ once. Meanwhile, the oxidized primary electron donor $\mathrm{P}$ is rereduced by accepting an electron from cytochrome $b c_{1}$ at the periplasmic side of the protein. The RCs can be excited again and $\mathrm{Q}_{\mathrm{A}}$ can give a second electron to $\mathrm{Q}_{\mathrm{B}}$. The doubly reduced and protonated $\mathrm{Q}_{\mathrm{B}}$ leaves the $\mathrm{RCs}$ to the ubiquinone pool. New ubiquinone from the ubiquinone pool of the membrane replaces the ubiquinol leading to the intial state of the RCs (Blankenship 2002).

The selective isotopic labelling in RCs were achieved by using selective labelling of $\delta$-Aminolevulinic acid (ALA) which is a precursor for formation BChl and BPhe (Jordan 1991). Therefore by enriching the medium with $\left(3-{ }^{13} \mathrm{C}\right)$-ALA labelled precursor, specifically labelled RCs were produced, isolated and finally quinones were depleted (Fig. 2).

\section{Applications}

Photo-CIDNP MAS NMR allows studying the structure of such photosynthetic RCs at their atomic level. The transfer of polarization from the enormous photo-CIDNP could be transferred via spin diffusion to the neighboring nuclei which are also labeled. Currently, various NMR experiments are being designed for transferring the polarization from the reaction center to nearby nuclei. Further nanosecond laser photo-CIDNP allows us to study the kinetics of the polarization buildup. This could give us the insight of the importance and influence of the hyperfine coupling, anisotropic chemical shift playing role in mechanism of such huge polarization production. 
Fig. 2 Bio-synthetic pathway for labelled BRC

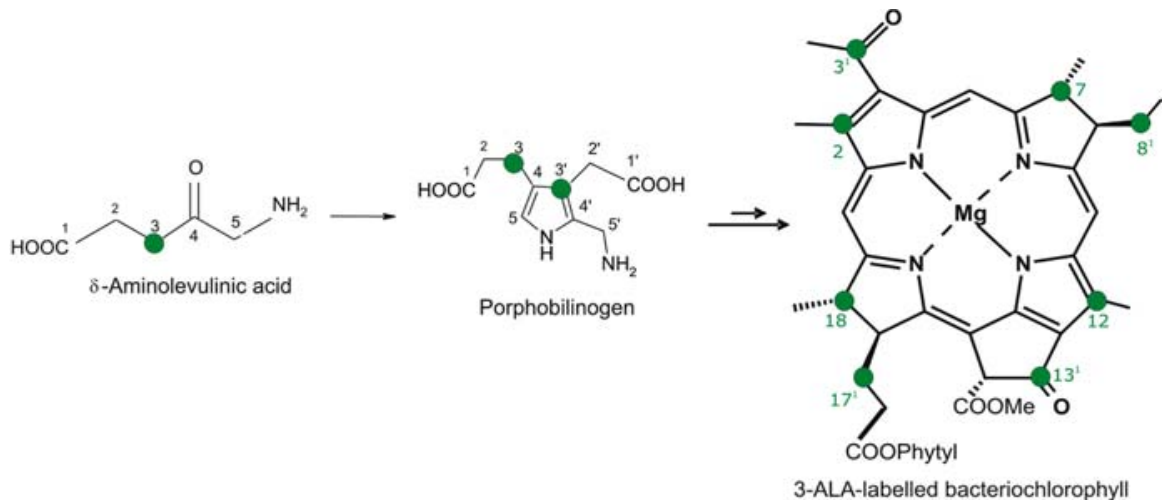

Currently, we find the photo-CIDNP works only on natural reaction centers from plants and bacteria. A schematic representation of the application of photo-CIDNP MAS NMR towards development of artificial RCs illustrated in Fig. 3. Hence if we can incorporate these on the surface of the big membrane protein then this could eventually lead us to find the structure and functionality of the surface of the big protein, otherwise very difficult to determine the structure as they can't be crystallized. Studying the photosynthetic bacteria by photo-CIDNP MAS NMR experiments opens the possibilities to understand the chemical environment required to make an artificial reaction center. During the photosynthesis, in RCs from cytochrome $\mathrm{bc}_{1}$ comples, cytochorome $b$ and cytochorome $c_{1}$ units produce +280 and $+285 \mathrm{mV}$ respectively versus NHE. In future these findings could eventually replace bacteria with an artificial chemical which then would produce energy just with the natural sunlight and water. The yields are low, but if one could eventually increase the efficiency, then this could answer the future energy problems.

\section{EMBO towards training of young researchers}

European Molecular Biology Organization (EMBO) is one of the funding agencies which aim not only promoting science but also giving opportunities to the young researchers to apply for various research grants. Fortunately they also offer short-term fellowships especially for the students who are doing their $\mathrm{PhD}$. Such fellowships encourages to students to

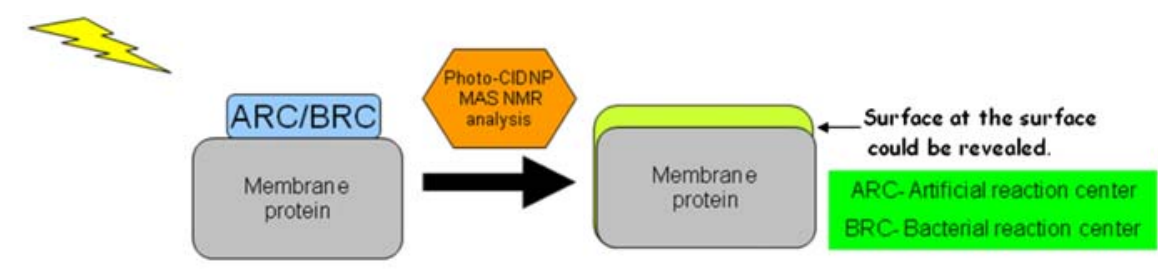

Schematic presentation of a Spin Torch

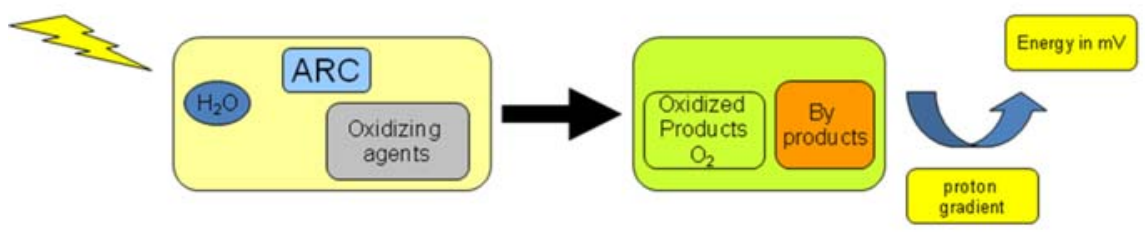

Scheme of generation of energy by ARC

Fig. 3 Futuristic applications of photo-CIDNP MAS NMR 
pursue their career in science towards successfully carrying out their dream project. I am very grateful to EMBO for this short-term grant to make it possible to proceed further. This also allowed me to visit ETH Zurich and collaborate with Prof. Jeschke and Dr. Matthias to learn more of the simulation of our experimental NMR data for better understanding and analysis.

Project Coordinator: Dr. Jöerg Matysik, Asst. Professor, Leiden Institute of Chemistry, University of Leiden.

\section{Collaborators:}

Prof. Dr. G. Jeschke, Physical Chemistry, ETH Zürich, Switzerland.

Dr. Matthias Ernst, PD, Physical Chemistry, ETH Zürich, Switzerland.

Open Access This article is distributed under the terms of the Creative Commons Attribution Noncommercial License which permits any noncommercial use, distribution, and reproduction in any medium, provided the original author(s) and source are credited.

\section{References}

Baldus M (2007) Biological solid-state NMR, methods and applications. J Biomol NMR 39:73-86

Blankenship R (2002) Molecular mechanism of photosynthesis. Blackwell, London

Dimitrios GV, Eleftherios T, Pietro M, Demetrios FG, Maria TG, David CC (2008) Development of a photosystem IIbased optical microfluidic sensor for herbicide detection. Talanta 77(1):42-47

Jeschke G, Matysik J (2003) A reassessment of the origin of photochemically induced dynamic nuclear polarization effects in solids. Chem Phys 294:239

Jordan PM (1991) Biosynthesis of tetrapyrroles. Elsevier, Amsterdam

Prakash S, Alia GP, de Groot HJM, Jeschke G, Matysik J (2005) Magnetic field dependence of photo-CIDNP MAS NMR on photosynthetic reaction centers of Rhodobacter Sphaeroides WT. J Am Chem Soc 127:14290-14298 